\title{
Personalized upper limb training combined with anodal-tDCS for sensorimotor recovery in spastic hemiparesis: study protocol for a randomized controlled trial
}

Mindy F. Levin ${ }^{1,2,8^{*}}$, Melanie C. Baniña ${ }^{1,2}$, Silvi Frenkel-Toledo ${ }^{3,4}$, Sigal Berman ${ }^{5}$, Nachum Soroker ${ }^{4,6}$, John M. Solomon ${ }^{7}$ and Dario G. Liebermann ${ }^{3}$

\begin{abstract}
Background: Recovery of voluntary movement is a main rehabilitation goal. Efforts to identify effective upper limb (UL) interventions after stroke have been unsatisfactory. This study includes personalized impairment-based UL reaching training in virtual reality (VR) combined with non-invasive brain stimulation to enhance motor learning. The approach is guided by limiting reaching training to the angular zone in which active control is preserved ("active control zone") after identification of a "spasticity zone". Anodal transcranial direct current stimulation (a-tDCS) is used to facilitate activation of the affected hemisphere and enhance inter-hemispheric balance. The purpose of the study is to investigate the effectiveness of personalized reaching training, with and without a-tDCS, to increase the range of active elbow control and improve UL function.

Methods: This single-blind randomized controlled trial will take place at four academic rehabilitation centers in Canada, India and Israel. The intervention involves 10 days of personalized VR reaching training with both groups receiving the same intensity of treatment. Participants with sub-acute stroke aged 25 to 80 years with elbow spasticity will be randomized to one of three groups: personalized training (reaching within individually determined active control zones) with a-tDCS (group 1) or sham-tDCS (group 2), or non-personalized training (reaching regardless of active control zones) with a-tDCS (group 3). A baseline assessment will be performed at randomization and two followup assessments will occur at the end of the intervention and at 1 month post intervention. Main outcomes are elbowflexor spatial threshold and ratio of spasticity zone to full elbow-extension range. Secondary outcomes include the Modified Ashworth Scale, Fugl-Meyer Assessment, Streamlined Wolf Motor Function Test and UL kinematics during a standardized reach-to-grasp task.
\end{abstract}

Discussion: This study will provide evidence on the effectiveness of personalized treatment on spasticity and UL motor ability and feasibility of using low-cost interventions in low-to-middle-income countries.

Trial registration: ClinicalTrials.gov, ID: NCT02725853. Initially registered on 12 January 2016.

Keywords: Stroke, Spasticity, Spatial threshold, tDCS, Neurorehabilitation

\footnotetext{
* Correspondence: mindy.levin@mcgill.ca

${ }^{1}$ School of Physical and Occupational Therapy, Faculty of Medicine, McGill

University, Montreal, QC, Canada

${ }^{2}$ Center for Interdisciplinary Research in Rehabilitation (CRIR), Montreal, QC,

Canada

Full list of author information is available at the end of the article
} 


\section{Background}

Stroke is a leading cause of long-term disability. Up to $85 \%$ of patients with sub-acute stroke present chronic upper limb (UL) sensorimotor deficits [1]. While poststroke UL recovery has been a major focus of attention, efforts to identify effective rehabilitation interventions have been unsatisfactory. This study focuses on the delivery of personalized impairment-based UL training combined with low-cost state-of-the-art technology (non-invasive brain stimulation and commercially available virtual reality, VR) to enhance motor learning, which is becoming more readily available worldwide.

A major impairment following stroke is spasticity, leading to difficulty in daily activities and reduced quality of life [2]. Studies have identified that spasticity relates to disordered motor control due to deficits in the ability of the central nervous system to regulate motoneuronal thresholds through segmental and descending systems [3, 4]. In the healthy nervous system, the motoneuronal threshold is expressed as the "spatial threshold" (ST) or the specific muscle length/joint angle at which the stretch reflex and other proprioceptive reflexes begin to act [5-7]. The range of ST regulation in the intact system is defined by the task-specific ability to activate muscles anywhere within the biomechanical joint range of motion (ROM). However, to relax the muscle completely, ST has to be shifted outside of the biomechanical range [8].

After stroke, the ability to regulate STs is impaired [3] such that the upper angular limit of ST regulation occurs within the biomechanical range of the joint resulting in spasticity (spasticity zone). Thus, resistance to stretch of the relaxed muscle has a spatial aspect in that it occurs within the defined spasticity zone. In other joint ranges, spasticity is not present and normal reciprocal muscle activation can occur (active control zone; [4] Fig. 1). This theory-based intervention investigates whether recovery of voluntary movement is linked to recovery of ST control.

We also consider that inter-hemispheric balance is disrupted after stroke, interfering with recovery. UL motor function depends on the modulation of interhemispheric inhibition between cortical areas via transcallosal projections $[9,10]$ and descending projections to fingers, hand and arm [11]. Unilateral hemispheric damage reduces activity in the affected hemisphere while activity in the unaffected hemisphere increases [12], becoming more dominant. UL recovery may relate to rebalancing of inter-hemispheric inhibition [13] using, for example, anodal transcranial direct current stimulation (a-tDCS) over the affected hemisphere $[14,15]$. a-tDCS is considered a safe technique with transient adverse effects, such as slight scalp itching or tingling and/or mild headaches, that are not expected to impede the patient's ability to participate in the training protocol [16].

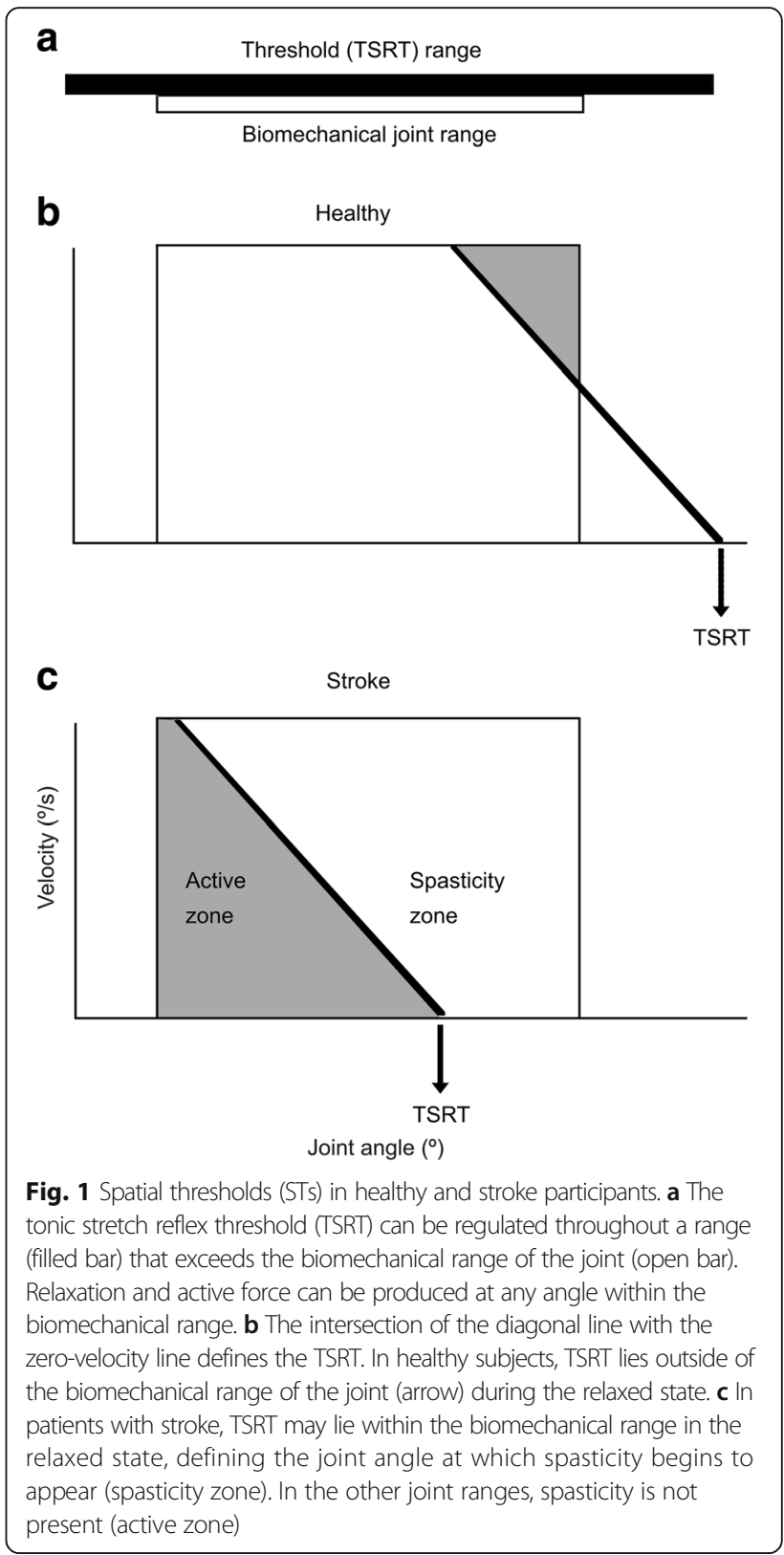

The underlying idea of this proposal is that recovery of voluntary movement is tightly linked to the recovery of threshold control. We propose an intervention that combines current knowledge about motor learning and disorders in ST control. The intervention involves personalized UL reach training designed according to the spatial structure of motor deficits of an individual, with excitatory a-tDCS over the sensorimotor areas of the affected hemisphere.

\section{Methods}

Aim, design and setting

We hypothesize that in patients with sub-acute spastic hemiparesis after stroke, practice restricted to the active 
control zone (personalized training) combined with excitatory a-tDCS will improve control over the range of ST regulation in the elbow and improve UL kinematic and clinical performance measures compared to nonpersonalized training with a-tDCS and personalized training with sham-tDCS. We propose a single-blind, parallel, three-group, randomized controlled trial (RCT; allocation ratio 1:1:1) involving personalized therapeutic intervention to improve control over the range of regulation of STs with the application of excitatory a-tDCS, which, under generally facilitatory conditions (e.g., motivating patients with VR games), may strengthen remaining connections and help restore inter-hemispheric balance. The Standard Protocol Items: Recommendations for Interventional Trials (SPIRIT) flow diagram of study enrollment, interventions and assessments is shown in Fig. 2. In addition, the implementation of such training programs in a low-to-middle-income country will be investigated by including four academic rehabilitation centers in Canada, Israel and India.

\section{Participants and recruitment}

Sixty people with sub-acute spastic hemiparesis after stroke will be recruited during their hospitalization in rehabilitation centers. In order to achieve adequate participant enrollment, constant chart review of newly admitted patients will be done. Individuals will be included if they have/are: (1) a stroke in the middle cerebral territory, confirmed by magnetic resonance imaging/computed tomography (MRI/ CT), and medically stable; (2) aged 25-80 years; (3) in the sub-acute stage of stroke ( 3 weeks to 6 months post stroke); (4) arm paresis (2-6/7 on the Chedoke-McMaster Stroke Assessment arm score, CMSA [17]) and able to perform at least $30^{\circ}$ elbow flexion and extension; (5) elbow flexor and/ or extensor spasticity ( $>1+$ out of 4 on the Modified Ashworth Scale, MAS [18, 19]); and (6) able to provide informed consent. Exclusion criteria are: (1) other major neurological or neuromuscular/orthopedic problems or pain interfering with results' interpretation; (2) medical comorbidities such as HIV/AIDS, etc.; (3) major cognitive deficits $(<20$ on the Montreal Cognitive Assessment, MoCA [20]); (4) history of psychiatric disorders, alcohol or drug abuse, seizures, migraines, metal in the cranium, and cochlear and cardiac implants; (5) taking medications (e.g., anti-epileptic and psychoactive drugs) that could affect brain activity [21, 22]. Criteria for discontinuation include participant request or adverse events.

Eligibility will be established by a blinded research coordinator. Participants will provide informed consent and be allocated to one of three treatment groups $(n=$ 20 per group) by permuted block randomization, balanced for age (25-50 years/51-80 years) and chronicity (3-12 weeks/13-24 weeks). Participants will receive a training and evaluation schedule, and participation will be monitored by the study coordinator, who will also provide reminders to attend scheduled activities. Travel expenses will be remunerated for follow-up visits.

The sample size is based on preliminary data showing that the mean change in elbow-flexor ST angle was $10^{\circ}$ in stroke subjects with spasticity who underwent 2 weeks of transcutaneous electrical nerve stimulation treatment compared to $2^{\circ}$ after sham stimulation (unpublished data). Considering an $\alpha$ level of $5 \%$ and a $95 \%$ power (effect size $=2.23$ ) to detect differences using a mixed-design $3 \times 3$ analysis of variance (ANOVA) (G*Power 3.1.1), the minimal sample size is 13 subjects per group. Sample size was increased to 18 per group to have an equal number of

\begin{tabular}{|c|c|c|c|c|c|}
\hline & \multicolumn{5}{|c|}{ STUDY PERIOD } \\
\hline & Enrolment & Allocation & Post-alloc & ation & Follow up test \\
\hline TIMEPOINT & $-t_{1}$ & $\mathbf{0}$ & Intervention $\left(t_{1-10}\right)$ & Post-test $\left(t_{11}\right)$ & $t_{12}$ \\
\hline \multicolumn{6}{|l|}{ ENROLMENT: } \\
\hline \multirow{3}{*}{$\begin{array}{r}\text { Eligibility screen } \\
\text { Informed consent } \\
\text { Allocation }\end{array}$} & $\mathrm{X}$ & & & & \\
\hline & $\mathrm{X}$ & & & & \\
\hline & & $\mathrm{X}$ & & & \\
\hline \multicolumn{6}{|l|}{ INTERVENTIONS: } \\
\hline \multirow{2}{*}{$\begin{array}{l}\text { Group 1 } \\
\text { Group } 2\end{array}$} & & & $\mathrm{X}$ & & \\
\hline & & & $\mathrm{X}$ & & \\
\hline Group 3 & & & $\mathrm{X}$ & & \\
\hline \multicolumn{6}{|l|}{ ASSESSMENTS: } \\
\hline Baseline variables: CMSA, MoCA, HADS, EHI & $\mathrm{X}$ & & & & \\
\hline Outcome variables: MAS, FMA, WMFT & $\mathrm{X}$ & & & $\mathrm{X}$ & $\mathrm{X}$ \\
\hline
\end{tabular}

Fig. 2 Schedule of enrollment, interventions and assessments. Standard Protocol Items: Recommendations for Interventional Trials (SPIRIT) flow diagram of study enrollment, interventions and assessments 
subjects per group per site (six per group, per site). We will recruit 20 subjects per country overall considering a dropout rate of $10-15 \%$ for a final cohort of 60 subjects.

Prior to the start of recruitment of participants, standardized procedures will be established to ensure consistency across sites. Intervention therapists will receive training guidelines and evaluators will receive screening and clinical assessment documents. In addition, all team members directly involved in data collection will have face-to-face training on data collection protocols. Quarterly online meetings will be scheduled to maintain adherence to the protocols across sites.

\section{Intervention}

Participants will enroll in a 10-day training program over two consecutive weeks (5 days per week). Outcome evaluators, statistical analysts and care providers will be blind to group assignment but not the intervention therapists, given the nature of the intervention. Intervention therapists will receive allocation information from the study coordinator via coded email. Participants will be randomly allocated as follows: (group 1) personalized training (reaching within determined active control zones) and a-tDCS over the affected hemisphere; (group 2) non-personalized training (reaching in both spasticity and active control zones; full active ROM) and a-tDCS over the affected hemisphere; and (group 3) personalized training and sham-tDCS.

Prior to the reaching training, elbow-flexor STs in the hemiparetic arm will be determined in each participant to identify the active control zone, using the Montreal Stretch Reflex Threshold device (MSRT; [23] see below). For the personalized training groups (groups 1 and 3), elbow extension will be restricted to the active control range using a range-restriction elbow brace (Breg Inc., Carlsbad, CA, USA) blocking elbow extension beyond the permitted ST. Participants in the non-personalized training group will wear the brace, but elbow ROM will not be restricted. All participants will have partial arm-weight support with a sling during the training to avoid fatigue. Since a goal of the training is to increase isolated elbow extension, the sling will be placed so that the weight of the upper arm and shoulder is supported without restricting elbow movements. The support will be provided throughout the training sessions for all subjects so that training parameters are equivalent across groups and sites.

During reaching training, participants will sit on an armless chair and make discrete arm reaches towards targets displayed at various locations in the threedimensional (3D) workspace with unrestrained trunk movement. Training will be guided by a physical or occupational therapist for $50 \mathrm{~min}$ (actual movement time) per day. Four interactive games from the Jintronix VR system (Montreal, QC, Canada) will be viewed on a large screen. Three activities will involve unimanual movement and one will involve a bimanual activity (Fig. 3). The total amount of movement time each training day will be recorded by the Jintronix system. Treatments will be matched for both duration and intensity across sites.

\section{Calibration and game progression}

In the personalized practice group, the Jintronix system will be calibrated prior to each training session according to the individual's ability to reach within the active zone only, whereas in the non-personalized practice group the calibration will be done based on the individual's full active ROM. In this way, the calibration procedure is used to set and progress the sagittal reaching distance in each game from near to far according to the length of the subject's effective sagittal reaching distance. Success in each reaching task depends on a combination of reaching distance, speed and precision according to Fitts' law [24]. Thus, difficulty levels of each activity will be increased by altering the location of the virtual targets and by decreasing the allowable movement time to complete each reach (i.e., by increasing movement speed), while target size remains the same. Levels will be progressed when the participant reaches at least 95\% success rate in three rounds. Game difficulty will be progressed according to the Challenge Point Theory of motor learning [25]. This theory suggests that learning is enhanced by optimally challenging the individual through manipulation of task difficulty according to their motor skill level and cognitive capacity. Since learning is also affected by performance-related feedback, participants will receive concomitant visual and auditory Knowledge of Results about task success when movements are made accurately and within a specified lapse of time programmed by the therapist. Participants will also receive negative Knowledge of Performance about the use of compensatory trunk movements during reaching through the Jintronix system. Thus, if the participant leans their trunk more than $5^{\circ}$ in the sagittal direction, a yellow square will appear on the screen and a reach success will not be registered. The $5^{\circ}$ trunk-flexion range was chosen since it corresponds to the cutoff value identified in Subramanian et al. [26] as indicating compensatory trunk movement. Regardless of the training zone and difficulty level, all participants in each group will follow the same training guidelines and complete the $50 \mathrm{~min}$ of active movement time.

A-tDCS (Soterix, New York, NY, USA) will be provided by a constant direct current stimulator at an intensity of $1.5 \mathrm{~mA}$, applied via saline-soaked surface electrodes $(5 \mathrm{~cm} \times 7 \mathrm{~cm})$ on the scalp. Stimulation will occur during the initial $30 \mathrm{~min}$ of the 50 -min training session. The anode will be placed over the sensorimotor areas of the affected hemisphere (placed over $\mathrm{C} 3 / \mathrm{C} 4$ 

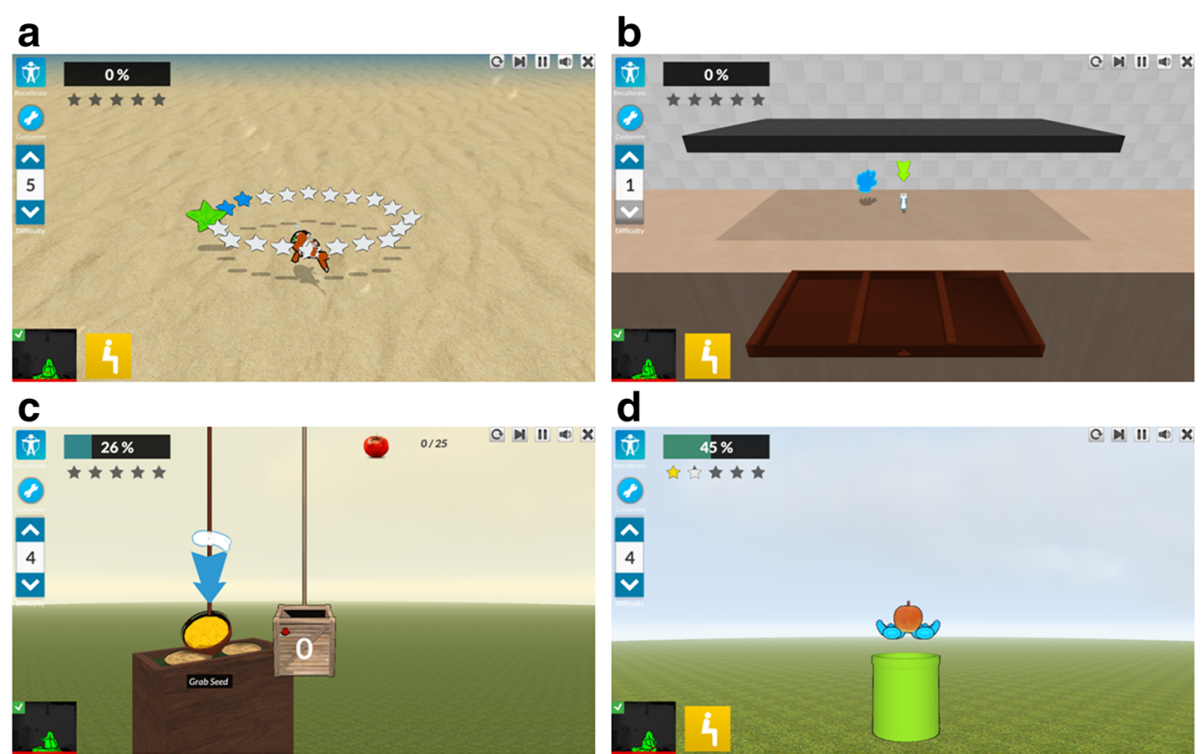

Fig. 3 Jintronix virtual reality (VR) games used in the intervention. a Fish Frenzy game requires the player to trace a three-dimensional (3D) trajectory by moving a fish on the screen in different shapes. $\mathbf{b}$ Kitchen Cleanup game requires forward reaching towards kitchen cutlery and returning them to shelves and drawers. c Garden Grab game requires lateral reaching while planting seeds, harvesting and transferring tomatoes to baskets. d Catch, Carry, Drop game requires bilateral coordination while catching apples, carrying and dropping them into a container

using the electroencephalogram (EEG) 10-20 referencing system), while the cathode will be placed on the contralateral supraorbital area. For the sham stimulation, the same electrode placement will be followed, but the device pre-programmed sham setting will apply stimulation only for the first minute. In the first $30 \mathrm{~s}$, a stimulation ramp-up is expected to cause a mild itching sensation underneath the anodal electrode. This sensation is expected to fade between 40 and $60 \mathrm{~s}$, at the end of which the intensity reaches zero level. No stimulation will be provided for the rest of the practice session.

As this trial intervention is carried out in different centers, the adherence to the intervention will be ensured by recording the frequency and the intensity of the games on standardized documents, supervision by the lead investigator at each site and supervision by the co-principal investigators (PIs) via regular meetings.

\section{Study outcomes}

Outcome measures will be documented before (Pre), after 10 days of intervention (Post) and 1 month after intervention end (Follow-up). Measures will be used to obtain an overall clinical profile of arm and hand impairment and function. Arm motor recovery will be evaluated at two levels of the International Classification of Function: Body Structure and Function (motor impairment) and Activity [27]. The primary outcome will be the ST angle and the ratio between the range of the biceps spasticity zone and the full biomechanical elbowextension ROM.

\section{Assessment of spasticity and active control zones}

Since active elbow extension (active control zone) is limited by the location of the ST in the angular range of the elbow, the ST angle of the elbow flexors (biceps brachii, $\mathrm{BB})$ will be measured. This will allow us to determine the range of angles in which $\mathrm{BB}$ cannot be relaxed (spasticity zone) and the range in which reciprocal agonist/antagonist muscle activation is possible (active control zone). The ST angle will be defined using the MSRT, a portable clinical device consisting of a two-channel electromyography (EMG) system (Procomp 5, Thought Technology, Montreal, QC, Canada), an electrogoniometer (servo-type rotational-position potentiometer P2200; Novotechnik U.S. Inc., Southborough, MA, USA) and dedicated software implemented on a laptop computer (Fig. 4). The MSRT has moderate-to-good intra- and inter-evaluator reliability for the measurement of elbow spasticity in poststroke participants [23].

$\mathrm{BB}$ and triceps brachii (TB) EMG recordings will be obtained with surface electrodes (Ambu ${ }^{\oplus}$ Blue Sensor P, Ballerup, Denmark), from motor points indicated in the Surface ElectroMyoGraphy for the Non-Invasive Assessment of Muscles (SENIAM) guidelines [28]. EMG signals will be band-pass filtered $(10-1000 \mathrm{~Hz})$, amplified $(\times 500)$ and sampled at 2048 samples/s. Seated participants will perform a maximal voluntary contraction of the elbow flexors to adjust EMG gain and initial elbow angle will be specified. Then, 20 stretches will be performed manually by stretching the elbow from flexion (approximately $50^{\circ}$ ) to full extension. Participants will be instructed to relax 


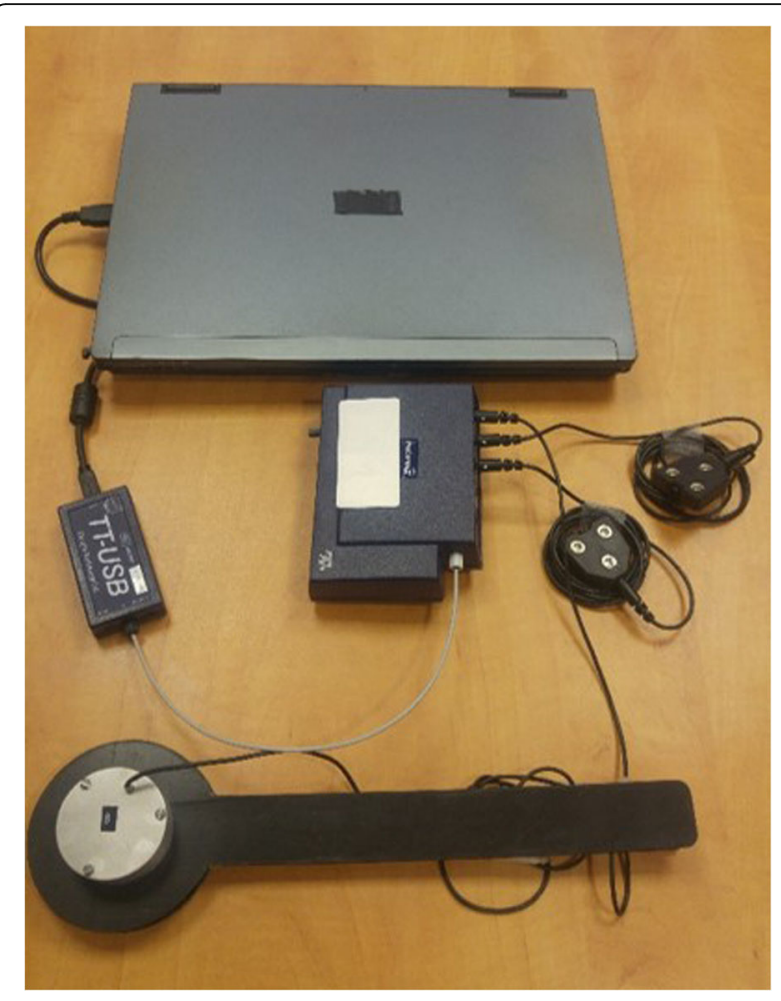

Fig. 4 Montreal Stretch Reflex Threshold (MSRT) device. The MSRT is a portable, clinical device consisting of a two-channel electromyography (EMG) system, an electrogoniometer and dedicated software implemented on a laptop computer

completely without assisting or resisting the angular displacement. Adequate rest (10 s) will be allowed between stretches to minimize effects of fatigue and muscle thixotropy [29]. Before each stretch, a randomly assigned speed, equally distributed between slow, moderate and fast velocities, will be indicated by an auditory signal from the MSRT to avoid the participant anticipating the upcoming stretch. Each evaluation will last for approximately $20 \mathrm{~min}$. At the end of each stretch, MSRT identifies the angle at which the EMG begins, called the dynamic stretch reflex threshold (DSRT) [23]. At the completion of at least 20 correct stretches, a linear regression line is computed through the DSRTs on the angular velocity/displacement plot from which the correlation coefficient $\left(r^{2}\right)$, slope and $x$-axis intercept are determined. The $\mathrm{x}$-axis intercept corresponds to the tonic stretch reflex threshold (TSRT) angle. A low TSRT angle corresponds to a high level of spasticity.

\section{Arm motor impairment}

Voluntary arm motor ability and coordination will be evaluated using the valid and reliable Fugl-Meyer Assessment (FMA) [30-32] that evaluates reflexes, volitional movements and rapid alternating movements. UL scores range from 0 to 66. Sensation (light touch), kinesthesia and passive ROM will be evaluated using FMA scales.
Clinical spasticity in elbow flexors and extensors will be measured with the MAS $[18,19]$ on six-point ordinal scales $(0-1,1+, 2-4)$. The MAS has poor-to-good interrater reliability $[19,33,34]$.

\section{Arm motor function}

Arm functional ability will be assessed with the six-item Streamlined Wolf Motor Function Test (S-WMFT) consisting of (1) speed of completion and (2) movement quality scores. Items 1 and 2 are activities involving elbow and shoulder movements and items 3 to 6 are functional tasks. Each item has a maximal time of $120 \mathrm{~s}$ and the mean will be reported. In the case of inability to complete a task, a time score of $121 \mathrm{~s}$ will be assigned [35]. Functional ability is rated on a six-point scale from 0 to 5 where 0 indicates no attempt to move the affected $\mathrm{UL}$ and 5 indicates normal movement. Mean scores will be reported. The S-WMFT has good concurrent (Spearman's $\rho=0.69$ ) and predictive validity (Spearman's $\rho=$ 0.68) with FMA. Responsiveness, as assessed by the standard response mean, was 0.41 [36].

\section{Kinematic recording and analysis of reach-to-grasp task}

A kinematic assessment of a common daily reach-tograsp task requiring different amounts of elbow extension will be recorded with a room- and body-calibrated five-sensor wireless electromagnetic tracking system G4 (Polhemus, Colchester, VT, USA; RMS static accuracy $0.20 \mathrm{~cm}$ for position and $0.5^{\circ}$ for orientation at a distance of $1 \mathrm{~m}$ from the transmitter). Each sensor is tracked at a rate of $120 \mathrm{~Hz}$ providing six degrees of freedom (three Cartesian and three polar). Sensors will be placed on the index finger metacarpo-phalangeal joint, the proximal third of the dorsal forearm, the mid-lateral surface of the arm, the mid-point of the superior-lateral border of the acromion, and mid-sternum.

Participants will sit in front of a table on a standard chair with no armrests. Arm position will be adjusted so that in the initial position the elbow will be in $30^{\circ}$ flexion alongside the body and feet will be supported on the floor. Participants will be instructed to reach to grasp a 6-cm diameter cone "as fast and as precisely as possible," hold or touch the cone for $2 \mathrm{~s}$, lift the cone or arm towards the chin and then return it to the table. A midsagittal reference frame will define four target locations. Targets 1 and 2 will be placed at two thirds and full arm's length in the mid-sagittal plane, respectively, for which different levels of elbow extension are required. Targets 3 and 4 will be placed at approximately $20 \mathrm{~cm}$ to the right and left of Target 2 . Arm length will be measured from the medial axillary border to the distal wrist crease with the elbow extended [37]. After several initial practice trials (two trials/target), participants will perform two sets of 40 trials $(2 \times 10$ trials $\times 4$ targets $=80$ 
movements; randomized), in approximately $1 \mathrm{~h}$. Rest between sets and trials will be provided.

Motor behavior will be measured at two levels: endpoint performance and arm movement quality [38]. Endpoint performance includes peak speed; trajectory straightness (index of curvature, IC) [39] where IC > 1.57 describes a semi-circle; and smoothness calculated as the number of velocity peaks in the tangential velocity profile.

For movement quality, joint angles involved in reaching will be measured including shoulder flexion, abduction/adduction and rotation, elbow extension, wrist supination and extension using Euler angles based on standard 3D kinematic reconstruction [40]. Analysis of inter-joint coordination, compensations and pathological synergies will include consideration of trunk and arm plane motion [41].

\section{Lesion type, size and location}

In a secondary analysis, the relationship between specific lesion type, size and location and treatment outcomes will be assessed.

\section{Data management}

The Oversight Committee will consist of the PIs and one other individual not associated with the trial. The Data Monitoring and Management Committee will be led by an individual who will ensure that all sites upload data from each phase of the trial to a secured, online data repository. Clinical, kinematic and EMG data will be coded for confidentiality. Adverse events due to atDCS, training or testing paradigms will be reported to the Ethics Committee at each site by filling out an incident report form. The Ethics Committee will then meet to discuss possible solutions.

\section{Statistical analyses}

Descriptive statistics will be used to highlight main demographic and clinical characteristics of participants. After verification for linearity, normality and homoscedasticity, parametric mixed-model ANOVAs will be used to determine which stimulation and practice modality combinations lead to greater improvements. The mixed-model analysis will include one between-subject factor - group with three levels (personalized training + a-tDCS, nonpersonalized training $+\mathrm{a}-\mathrm{tDCS}$ and personalized training + sham-tDCS), and one within-subject factor - time with three levels (Pre, Post and Follow-up). Multiple linear regression analyses on pooled data will identify relationships between primary outcomes and changes in clinical and kinematic measures. If assumptions for parametric tests are not met, non-parametric tests will be substituted. Results will be analyzed based on intention-to-treat principles. Individual participation in other in-patient services will be documented (physiotherapy, occupational therapy, speech therapy, social work, recreation, other) and compared between sites to account for effects of usual-care exposure. Results will be disseminated to individuals, user groups, health care and research professionals at trial completion.

\section{SPIRIT Checklist}

Please see Additional file 1 for the SPIRIT Checklist [42].

\section{Discussion}

Based on the identification of disorders in stretch reflex threshold control in each subject, we will implement a personalized VR-enhanced UL training program with and without a-tDCS. Our innovative approach aims to maximize the recovery potential of each individual by taking into consideration motor impairment as defined by Threshold Control Theory [8] and training based on established principles of motor learning that are assumed to maximize neural plasticity. Optimization of UL motor function is expected due to timing the intervention during the sub-acute phase of stroke when the most rapid recovery occurs [43].

During the design phase of the RCT, low-cost equipment was specifically chosen to enable accessibility for institutions in any country regardless of income level. To date, all centres successfully built the study set-up and simultaneously started implementation of the study.

\section{Trial status}

The RCT recruitment is planned from June 2016 to December 2018. Data analysis and evaluation will be performed subsequently. Final results of this study will be published.

\section{Additional file}

Additional file 1: SPIRIT 2013 Checklist: recommended items to address in a clinical trial protocol and related documents. (PDF $152 \mathrm{~kb}$ )

\section{Abbreviations}

a-tDCS: Anodal transcranial direct current stimulation; BB: Biceps brachii; CMSA: Chedoke-McMaster Stroke Assessment; CT: Computed tomography; DSRT: Dynamic stretch reflex threshold; EEG: Electroencephalogram; EMG: Electromyography; FMA: Fugl-Meyer Assessment; BUT: Modified Ashworth Scale; Mocha: Montreal Cognitive Assessment; MRl: Magnetic resonance imaging; MSRT: Montreal Stretch Reflex Threshold device; ROM: Range of motion; Senior: Surface ElectroMyoGraphy for the NonInvasive Assessment of Muscles; ST: Spatial threshold; S-WMFT: Streamlined Wolf Motor Function Test; TB: Triceps brachii; TSRT: Tonic stretch reflex threshold; UL: Upper limb; VR: Virtual reality

\section{Acknowledgements}

The authors acknowledge Rhona Guberek, Réjean Prévost, Fréderic

Méssier, Maureen MacMahon, Franceen Kaizer, Marie-Thérèse Laramée, Arel Shasha, Tal Galinka, Akash Shah and Subramanian Durairaj for their invaluable contributions to the success of this RCT.

\section{Funding}

This project is supported by the Canada-Israel Health Research Program (MFL and DGL), a program that is jointly funded by: Canadian Institutes of Health Research, Azrieli Foundation, International Development Research Center and Israel Science Foundation; IDRC grant number 108186-001, ISF grant number 2392\15 (IDRC, 
150 Kent St, Ottawa, ON K1P 0B2, tel: +613-236-6163). MCB is supported in part by the Zavalkoff Family Foundation's Brain @McGill Graduate/Postdoctoral Award.

\section{Availability of data and materials}

The datasets generated and/or analyzed during the current study will not be publicly available due to patient confidentiality rules, but anonymized data would be available from the corresponding author on reasonable request.

\section{Authors' contributions}

MCB and SFT equally assume primary authorship. MFL and DGL provided overall concept/research design. MCB performs the kinematic assessments, schedules the clinical assessments and training sessions, collaborates with research team clinicians to screen in-patients for eligibility at the Jewish Rehabilitation Hospital and the Institut de Réadaptation Gingras-Lindsay de Montréal and maintains the research database for the Canada site. SFT performs the kinematic assessments, supervises the clinical assessments and training sessions, screens in-patients for eligibility at the Loewenstein Rehabilitation Hospital, and maintains the research database for the Israel site. SB provided concept/research design, designed the data collection program for the kinematic assessment and implemented the study database. NS provided concept/research design, planned the lesion analysis and acts as primary physician investigator at the Loewenstein Rehabilitation Hospital. JMS provided concept/research design and supervises the kinematic, clinical and training sessions in Kasturba Medical Hospital in India. All authors contributed to the writing and approval of the final manuscript.

\section{Ethics approval and consent to participate}

Ethical approval was obtained from the appropriate ethics committees of each study site prior to the beginning of the study. The Centre de Recherche Interdisciplinaire en Réadaptation du Montréal Métropolitain (Montréal, QC, Canada) approved the study for the Jewish Rehabilitation Hospital and the Institut de Réadaptation Gingras-Lindsay de Montréal (CRIR1112-1115). The Institutional Ethics Review Board of Loewenstein Rehabilitation Hospital (Ra'anana, Israel) approved the study for the Loewenstein Rehabilitation Hospital (000-11-15-LOE) and the Institutional Ethics Committee of the Tel Aviv University (Tel Aviv, Israel). The Institutional Ethics Committee of Kasturba Hospital (Manipal, India) approved the study for the Kasturba Medical Hospital (IEC-32/2016). The trial was registered with the ClinicalTrials.gov registry in USA on 12 January 2016 with the trial registration number NCT02725853. The SPIRIT guidelines for implementation of study protocols were followed (Fig. 2 and Additional file 1) [42]. Written informed consent will be obtained from each participant.

Adverse effects will be documented and reported to the co-PIs. If adverse effects occur then participants will be withdrawn from their allocated treatment but included in the measurement battery according to the intention-to-treat principle. All protocol modifications will be registered with ClinicalTrials.gov.

\section{Consent for publication}

Not applicable. No individual data are contained in the manuscript.

\section{Competing interests}

Jintronix will provide licenses for use of their gaming system and technical support and servers. MFL holds a US patent for the MSRT analysis. The remaining authors declare that they have no competing interests or any conflicts of interest in the authorship or publication of this study.

\section{Publisher's Note}

Springer Nature remains neutral with regard to jurisdictional claims in published maps and institutional affiliations.

\section{Author details}

${ }^{1}$ School of Physical and Occupational Therapy, Faculty of Medicine, McGill University, Montreal, QC, Canada. ${ }^{2}$ Center for Interdisciplinary Research in Rehabilitation (CRIR), Montreal, QC, Canada. ${ }^{3}$ Department of Physical Therapy, Stanley Steyer School of Health Professions, Sackler Faculty of Medicine, Tel Aviv University, Tel Aviv, Israel. ${ }^{4}$ Department of Neurological Rehabilitation, Loewenstein Hospital, Ra'anana, Israel. ${ }^{5}$ Department of Industrial Engineering and Management, Ben-Gurion University of the Negev, Beer-Sheva, Israel. ${ }^{6}$ Sackler Faculty of Medicine, Tel Aviv University, Tel Aviv, Israel. ${ }^{7}$ Department of Physiotherapy, School of Allied Health Sciences (SOAHS), Manipal University, Manipal, Karnataka, India. ${ }^{8}$ School of Physical and Occupational
}

Therapy, McGill University, 3654 Promenade Sir William Osler, Montreal, QC H3S 1Y5, Canada.

Received: 14 February 2017 Accepted: 8 November 2017

Published online: 04 January 2018

\section{References}

1. Feigin $\mathrm{VL}$, Lawes $C M$, Bennett DA, et al. Worldwide stroke incidence and early case fatality reported in 56 population-based studies: a systematic review. Lancet Neurol. 2009;8(4):355-69.

2. Nichols-Larsen DS, Clark PC, Zeringue A, et al. Factors influencing stroke survivors' quality of life during subacute recovery. Stroke. 2005;36(7):1480-4.

3. Levin MF, Feldman AG. The role of stretch reflex threshold regulation in normal and impaired motor control. Brain Res. 1994;657(1-2):23-30.

4. Levin MF, Selles RW, Verheul MH, Meijer OG. Deficits in the coordination of agonist and antagonist muscles in stroke patients: implications for normal motor control. Brain Res. 2000;853(2):352-69.

5. Matthews PB. A study of certain factors influencing the stretch reflex of the decerebrate cat. J Physiol. 1959;147:547-64.

6. Asatryan DG, Fel'dman AG. Functional tuning of the nervous system with control of movement or maintenance of a steady posture - I. Biophysics. 1965;10:925-35.

7. Feldman AG, Orlovsky GN. The influence of different descending systems on the tonic stretch reflex in the cat. Exp Neurol. 1972;37:481-94.

8. Feldman AG. Space and time in the context of equilibrium point theory. Wiley Interdiscip Rev Cogn Sci. 2011;2:287-304.

9. Beaule $\mathrm{V}$, Tremblay $\mathrm{S}$, Theoret $\mathrm{H}$. Interhemispheric control of unilateral movement. Neural Plast. 2012;2012:1-11.

10. Duque J, Murase N, Celnik P, et al. Intermanual differences in movementrelated interhemispheric inhibition. J Cogn Neurosci. 2007;19:204-13.

11. Lemon RN. Descending pathways in motor control. Annu Rev Neurosci. 2008:31:195-218.

12. Takeuchi N, Oouchida Y, Izumi S-I. Motor control and neural plasticity through interhemispheric interactions. Neural Plast. 2012;2012:1-13.

13. Di Pino G, Pellegrino G, Assenza G, et al. Modulation of brain plasticity in stroke: a novel model for neurorehabilitation. Nat Rev Neurol. 2014; 10(10):597-608.

14. Allman C, Amadi U, Winkler AM, et al. Ipsilesional anodal tDCS enhances the functional benefits of rehabilitation in patients after stroke. Sci Transl Med. 2016;8(330):330re1.

15. Bastani A, Jaberzadeh S. Does anodal transcranial direct current stimulation enhance excitability of the motor cortex and motor function in healthy individuals and subjects with stroke: a systematic review and meta-analysis. Clin Neurophysiol. 2012;123(4):644-57.

16. Brunoni AR, Amadera J, Berbel B, et al. A systematic review on reporting and assessment of adverse effects associated with transcranial direct current stimulation. Int J Neuropsychopharmacol. 2011;14(8):1133-45.

17. Gowland C, Stratford P, Ward M, et al. Measuring physical impairment and disability with the Chedoke-McMaster Stroke Assessment. Stroke. 1993;24(1):58-63.

18. Ashworth B. Preliminary trial of carisoprodol in multiple sclerosis. Practitioner. 1964;192:540-2.

19. Bohannon RW, Smith MB. Interrater reliability of a modified Ashworth scale of muscle spasticity. Phys Ther. 1987;67:206-7.

20. Nasreddine ZS, Phillips NA, Bedirian V, et al. The Montreal Cognitive Assessment, MoCA: a brief screening tool for mild cognitive impairment. J Am Geriatr Soc. 2005;53(4):695-9.

21. Brunoni AR, Nitsche MA, Bolognini N, et al. Clinical research with transcranial direct current stimulation (tDCS): challenges and future directions. Brain Stimul. 2012;3:175-95.

22. Brunoni AR, Ferrucci $R$, Bortolomasi M, et al. Interactions between transcranial direct current stimulation (tDCS) and pharmacological interventions in the major depressive episode: findings from a naturalistic study. Eur Psychiatry. 2013;28(6):356-61.

23. Calota A, Feldman AG, Levin MF. Spasticity measurement based on tonic stretch reflex threshold in stroke using a portable device. Clin Neurophysiol. 2008;119(10):2329-37.

24. Fitts PM, Posner MI. Human performance. Belmont: Brooks/Cole; 1967.

25. Guadagnoli MA, Lee TD. Challenge point: a framework for conceptualizing the effects of various practice conditions in motor learning. J Mot Behav. 2004;36(2):212-24 
26. Subramanian SK, Yamanaka J, Chilingaryan G, Levin MF. Validity of movement quality kinematic variables as measures of arm motor impairment post-stroke. Stroke. 2010;41(10):2303-8.

27. World Health Organization. International classification of functioning, disability and health: ICF. World Health Organization; 2001.

28. Freriks B, Hermens H, Disselhorst-Klug C, Rau G. The recommendations for sensors and sensor placement procedures for surface electromyography. In: SENIAM 8: European recommendations for surface electromyography. Enschede: Roessingh Research and Development BV; 1999. p. 15-53.

29. Hagbarth KE, Hägglund JV, Nordin M, Wallin EU. Muscle thixotropy and its effects on spindle and reflex responses to stretch. In: Clinical aspects of sensory motor integration. Berlin: Springer-Ver; 1987. p. 91-7.

30. Fugl-Meyer AR, Jaasko L, Leyman I, et al. The post-stroke hemiplegic patient. 1. A method for evaluation of physical performance. Scand J Rehabil Med. 1975:7(1):13-31

31. Berglund $K$, Fugl-Meyer AR. Upper extremity function in hemiplegia. A cross validation study of two assessment methods. Scand J Rehabil Med. 1986;18:155-7.

32. Duncan PW, Propst M, Nelson SG. Reliability of the Fugl-Meyer assessment of sensorimotor recovery following cerebrovascular accident. Phys Ther. 1983;63(10):1606-10.

33. Blackburn $M$, van Vliet $P$, Mockett SP. Reliability of measurements obtained with the modified Ashworth scale in the lower extremities of people with stroke. Phys Ther. 2002;82:25-34.

34. Pandyan AD, Johnson GR, Price $\mathrm{Cl}$, et al. A review of the properties and limitations of the Ashworth and modified Ashworth Scales as measures of spasticity. Clin Rehabil. 1999;13:373-83.

35. Bogard K, Wolf S, Zhang Q, et al. Can the Wolf Motor Function Test be streamlined? Neurorehabil Neural Repair. 2009;23(5):422-8.

36. Wu C, Fu T, Lin KC, et al. Assessing the streamlined Wolf Motor Function Test as an outcome measure for stroke rehabilitation. Neurorehabil Neural Repair. 2011;25:194-9.

37. Schneiberg S, Sveistrup H, McFadyen B, et al. The development of coordination for reach-to- grasp movements in children. Exp Brain Res. 2002;146:142-54

38. Levin MF, Kleim JA, Wolf SL. What do motor "recovery" and "compensation" mean in patients following stroke? Neurorehabil Neural Repair. 2009;23(4):313-9.

39. Archambault $P$, Pigeon $P$, Feldman AG, et al. Recruitment and sequencing of different degrees of freedom during pointing movements involving the trunk in healthy and hemiparetic subjects. Exp Brain Res. 1999:126(1):55-67.

40. Biryukova EV, Roby-Brami A, Frolov AA, Mokhtari M. Kinematics of human arm reconstructed from spatial tracking system recordings. J Biomech. 2000; 33:985-95.

41. Merdler T, Liebermann DG, Levin MF, Berman S. Arm-plane representation of shoulder compensation during pointing movements in patients with stroke. J Electromyogr Kinesiol. 2013;23(4):938-47.

42. Chan AW, Tetzlaff JM, Gotzsche PC, Altman DG, Mann H, Berlin JA, Dickersin K, Hrobjartsson A, Schulz KF, Parulekar WR, et al. SPIRIT 2013 explanation and elaboration: guidance for protocols of clinical trials. BMJ. 2013;346: e7586.

43. Ward NS, Brown MM, Thompson AJ, Frackowiak RSJ. Neural correlates of motor recovery after stroke: a longitudinal fMRI study. Brain. 2003;126(11): 2476-96.

\section{Submit your next manuscript to BioMed Central and we will help you at every step:}

- We accept pre-submission inquiries

- Our selector tool helps you to find the most relevant journal

- We provide round the clock customer support

- Convenient online submission

- Thorough peer review

- Inclusion in PubMed and all major indexing services

- Maximum visibility for your research

Submit your manuscript at www.biomedcentral.com/submit

CBiomed Central 